\title{
Front Matter: Volume 10483
}

, "Front Matter: Volume 10483," Proc. SPIE 10483, Optical Coherence Tomography and Coherence Domain Optical Methods in Biomedicine XXII, 1048301 (5 April 2018); doi: 10.1117/12.2315746

SPIE. Event: SPIE BiOS, 2018, San Francisco, California, United States 


\section{PROGRESS IN BIOMEDICAL OPTICS AND IMAGING

\section{Optical Coherence Tomography and Coherence Domain Optical Methods in Biomedicine XXII}

Joseph A. Izatt

James G. Fujimoto

Valery V. Tuchin

Editors

29-31 January 2018

San Francisco, California, United States

Sponsored and Published by

SPIE 
The papers in this volume were part of the technical conference cited on the cover and title page. Papers were selected and subject to review by the editors and conference program committee. Some conference presentations may not be available for publication. Additional papers and presentation recordings may be available online in the SPIE Digital Library at SPIEDigitalLibrary.org.

The papers reflect the work and thoughts of the authors and are published herein as submitted. The publisher is not responsible for the validity of the information or for any outcomes resulting from reliance thereon.

Please use the following format to cite material from these proceedings:

Author(s), "Title of Paper," in Optical Coherence Tomography and Coherence Domain Optical Methods in Biomedicine XXII, edited by Joseph A. Izatt, James G. Fujimoto, Valery V. Tuchin, Proceedings of SPIE Vol. 10483 (SPIE, Bellingham, WA, 2018) Seven-digit Article CID Number.

ISSN: 1605-7422

ISSN: 1996-756X (electronic)

ISBN: 9781510614512

ISBN: 9781510614529 (electronic)

Published by

SPIE

P.O. Box 10, Bellingham, Washington $98227-0010$ USA

Telephone +1 3606763290 (Pacific Time) · Fax +1 3606471445

SPIE.org

Copyright (c) 2018, Society of Photo-Optical Instrumentation Engineers.

Copying of material in this book for internal or personal use, or for the internal or personal use of specific clients, beyond the fair use provisions granted by the U.S. Copyright Law is authorized by SPIE subject to payment of copying fees. The Transactional Reporting Service base fee for this volume is $\$ 18.00$ per article (or portion thereof), which should be paid directly to the Copyright Clearance Center (CCC), 222 Rosewood Drive, Danvers, MA 01923. Payment may also be made electronically through CCC Online at copyright.com. Other copying for republication, resale, advertising or promotion, or any form of systematic or multiple reproduction of any material in this book is prohibited except with permission in writing from the publisher. The CCC fee code is 1605$7422 / 18 / \$ 18.00$.

Printed in the United States of America.

Publication of record for individual papers is online in the SPIE Digital Library.

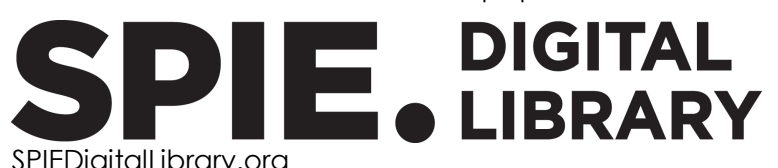

SPIEDigitalLibrary.org

Paper Numbering: Proceedings of SPIE follow an e-First publication model. A unique citation identifier (CID) number is assigned to each article at the time of publication. Utilization of CIDs allows articles to be fully citable as soon as they are published online, and connects the same identifier to all online and print versions of the publication. SPIE uses a seven-digit CID article numbering system structured as follows:

- The first five digits correspond to the SPIE volume number.

- The last two digits indicate publication order within the volume using a Base 36 numbering system employing both numerals and letters. These two-number sets start with $00,01,02,03$, 04, 05, 06, 07, 08, 09, OA, OB ... OZ, followed by 10-1Z, 20-2Z, etc. The CID Number appears on each page of the manuscript. 


\title{
Contents
}

\author{
$\checkmark$ Authors \\ vii Conference Committee
}

\section{OPHTHALMIC NEW TECHNOLOGY I}

1048306 Multimodal ophthalmic imaging using handheld spectrally encoded coherence tomography and reflectometry (SECTR) [10483-5]

\section{GI/ENDOSCOPIC/CATHETER}

$10483 \mathrm{OR}$ Automated detection of esophageal dysplasia in in vivo optical coherence tomography images of the human esophagus [10483-26]

\section{OPHTHALMIC NEW TECHNOLOGY II}

1048316 Resolution and throughput optimized intraoperative spectrally encoded coherence tomography and reflectometry (iSECTR) for multimodal imaging during ophthalmic microsurgery [10483-41]

1048319 Ultra-high speed OCT allows measurement of intraocular pressure, corneal geometry, and corneal stiffness using a single instrument [10483-45]

\section{OCT NEW TECHNOLOGY II}

$104831 \mathrm{~J}$ Spectral domain, common path OCT in a handheld PIC based system [10483-55]

NOVEL CONTRAST

1048326 Optical coherence tomography of lymphatic vessel endothelial hyaluronan receptors in vivo [10483-79]

$104832 B \quad$ Accurate estimation of the attenuation coefficient from axial point spread function corrected OCT scans of a single layer phantom [10483-84]

POSTER SESSION: TECHNOLOGY

$104832 \mathrm{C}$ Ophthalmic laser system integrated with speckle variance optical coherence tomography for real-time temperature monitoring [10483-44] 
10483 2D Speckle-modulation for speckle reduction in optical coherence tomography [10483-85]

$104832 \mathrm{~K}$ Snapshot polarization-sensitive plug-in optical module for a Fourier-domain optical coherence tomography system [10483-92]

$104832 \mathrm{~L}$ Time efficient Gabor fused master slave optical coherence tomography [10483-93]

$104832 \mathrm{M}$ Intra-retinal segmentation of optical coherence tomography images using active contours with a dynamic programming initialization and an adaptive weighting strategy [10483-94]

1048320 Non-destructive geometric and refractive index characterization of single and multielement lenses using optical coherence tomography [10483-96]

$104832 Q \quad$ Wavelet tree structure based speckle noise removal for optical coherence tomography [10483-98]

$104832 R \quad$ Wide field-of-view high lateral resolution optical coherence tomography with 3D image stitching and superresolution [10483-99]

1048325 Scaled nonuniform Fourier transform for image reconstruction in swept source optical coherence tomography [10483-100]

$104832 \mathrm{~T}$ Highly efficient superluminescent diodes and SLD-based combined light sources of red spectral range for applications in biomedical imaging [10483-101]

$104832 \mathrm{U}$ Automated intraretinal layer segmentation of optical coherence tomography images using graph-theoretical methods [10483-102]

$104832 \mathrm{~V}$ An OCT image denoising method based on fractional integral [10483-104]

POSTER SESSION: FUNCTIONAL AND APPLICATIONS

$104832 \mathrm{~W}$ Capillary red blood cell velocimetry by phase-resolved optical coherence tomography [10483-105]

$104832 Y$ Detection of brain tumor margins using optical coherence tomography [10483-107]

1048338 3D visualization of the ovarian tissue scattering coefficient with swept-source optical coherence tomography [10483-117]

1048339 Investigation in clinical potential of polarization sensitive optical coherence tomography in laryngeal tumor model study [10483-118] 


\section{Authors}

Numbers in the index correspond to the last two digits of the seven-digit citation identifier (CID) article numbering system used in Proceedings of SPIE. The first five digits reflect the volume number. Base 36 numbering is employed for the last two digits and indicates the order of articles within the volume. Numbers start with 00, 01, 02, 03, 04, 05, 06, 07, 08, 09, OA, OB...0Z, followed by 10-1Z, 20-2Z, etc.

Ahn, Yeh-Chan, 39

Andreeva, Ekaterina V., 2T

Anikeev, Andrei S., 2T

Baig, Sarfaraz, $2 R$

Boas, David A., $2 \mathrm{~W}$

Bradu, Adrian, 2K, $2 \mathrm{~L}$

Callewaert, Tom, 2B

Campos-Delgado, Daniel U., $2 Y$

Cernat, Ramona, 2L

Chamorovskiy, Alexander, 2T

Chen, Minghui, 2V

Cheon, Gyeongwoo, 2C

Chu, Steven, 2D

Dalal, Roopa, 26

de Boer, Johannes F., 2B

de la Zerda, Adam, 26, 2D

Dekker, Ronald, $1 \mathrm{~J}$

Dong, Jing, OR

Dutta, Rebecca, 26, 2D

El-Haddad, Mohamed T., 06, 16, 20

Erdener, Sefik Evren, 2W

Faber, Dirk J., $1 \mathrm{~J}$

Fernando, Namal, 25

Fu, Buyin, 2W

Ghafaryasl, Babak, 2B

Gholami, Peyman, 2M, 2 U

Hagemann, lan, 38

Han, Zhaolong, 19

$\mathrm{He}$, Jintao, 2V

Heideman, René G., $1 \mathrm{~J}$

Il'chenko, Stepan N., 2T

Jia, Wenyu, 2V

Jiang, Guomin, 2R

Jo, Dongki, $2 \mathrm{C}$

Jo, Javier, $2 Y$

Juarez-Chambi, Ronald M., $2 Y$

Kalkman, J., 2B

Kang, Jin U., $2 \mathrm{C}$

Kassinopoulos, Michalis, OR

Kim, Jongmin, 2C

Kim, Keun Bae, $1 \mathrm{~J}$

Kim, Kyungmin, $1 \mathrm{~J}$

Kim, Sung Won, 39

Kordi, Behzad, 25

Kuppuswamy Parthasarathy, Mohana, 2M, 2U

Kut, Carmen, $2 Y$

Lakshminarayanan, Vasudevan, $2 \mathrm{M}, 2 \mathrm{U}$

Larin, Kirill V., 19

Lee, Changho, $2 \mathrm{C}$

Lee, Jihoon, $2 \mathrm{C}$

Lee, Soohyun, 2C

Leeburg, Kelsey C., 06, 16

Leinse, Arne, $1 \mathrm{~J}$

Lew, Matthew D., 2D
Li, Xingde, $2 Y$

Liba, Orly, 26, 2D

Liu, Xuan, $2 Q$

Liv, Yang, $2 Q$

Malone, Joseph D., 06, 16

Marchenko, Denys, $1 \mathrm{~J}$

Marques, Manuel J., 2K

Mezgebo, Biniyam, 2S

Moshfeghi, Darius M., 2D

Nagib, Karim, 2S

Nair, Achuth, 19

Nandy, Sreyankar, 38

Oak, Chulho, 39

Ommani, Abbas, 2M

Pitris, Costas, OR

Podoleanu, Adrian, 2K, 2L

Powell, Matthew, 38

Qin, Xianfu, 2V

Quinones-Hinojosa, Alfredo, $2 Y$

Rao, Bin, 38

Rico-Jimenez, Jesus, $2 Y$

Rivet, Sylvain, 2K, 2L

Roy, Priyanka, $2 \mathrm{M}, 2 \mathrm{U}$

Ruis, Roosje M., $1 \mathrm{~J}$

Schill, Alexander, 19

Sen, Debasish, 26, 2D

Shen, Kai, $2 R$

Sherif, Sherif, $2 S$

Shidlovski, Vladimir R., 2T

Si, Peng, 26

Siegel, Cary, 38

Singh, Manmohan, 19

SoRelle, Elliott D., 2D

Tang, Jianbo, $2 \mathrm{~W}$

Tang, Shuo, 39

Tao, Yuankai K., 06, 16, 20

Tearney, Guillermo J., OR

Terrones, Benjamin D., 06, 16

Twa, Michael D., 19

van Leeuwen, Ton G., $1 \mathrm{~J}$

van Vliet, Lucas J., 2B

Vermeer, Koenraad A., 2B

Wang, Michael R., 2R

Wevers, Lennart, $1 \mathrm{~J}$

Winetraub, Yonatan, 26

Yakubovich, Sergei D., 2T

Yousefi, Siavash, 26

Yuan, Xin, 2Q

Zelek, John, 2M, 2U

Zeng, Yifeng, 38

Zheng, Gang, 2V

Zhou, Xin, 39

Zhu, Quing, 38 
Proc. of SPIE Vol. 10483 1048301-6

Downloaded From: https://www.spiedigitallibrary.org/conference-proceedings-of-spie on 26 Apr 2023 Terms of Use: https://www.spiedigitallibrary.org/terms-of-use 


\title{
Conference Committee
}

\author{
Symposium Chairs
}

James G. Fujimoto, Massachusetts Institute of Technology

(United States)

R. Rox Anderson, Wellman Center for Photomedicine, Massachusetts General Hospital (United States) and Harvard Medical School (United States)

Program Track Chairs

Tuan Vo Dinh, Fitzpatrick Institute for Photonics, Duke University (United States)

Anita Mahadevan-Jansen, Vanderbilt University (United States)

\section{Conference Chairs}

Joseph A. Izatt, Duke University (United States)

James G. Fujimoto, Massachusetts Institute of Technology (United States)

Valery V. Tuchin, N.G. Chernyshevsky Saratov National Research

State University (Russian Federation) and National Research Tomsk State University (Russian Federation) and Institute of Precision Mechanics and Control RAS (Russian Federation)

\section{Conference Program Committee}

Peter E. Andersen, Technical University of Denmark (Denmark)

Kostadinka Bizheva, University of Waterloo (Canada)

Stephen A. Boppart, University of Illinois at Urbana-Champaign (United States)

Zhongping Chen, Beckman Laser Institute and Medical Clinic (United States)

Johannes de Boer, Vrije University Amsterdam (Netherlands)

Wolfgang Drexler, Medizinische Universiteit Wien (Austria)

Grigory V. Gelikonov, Institute of Applied Physics (Russian Federation)

Christoph K. Hitzenberger, Medizinische Universiteit Wien (Austria)

Robert A. Huber, Universität zu Lübeck (Germany)

Rainer A. Leitgeb, Medizinische Universiteit Wien (Austria)

Xingde Li, Johns Hopkins University (United States)

Yingtian Pan, Stony Brook University (United States)

Adrian Gh. Podoleanu, University of Kent (United Kingdom)

Andrew M. Rollins, Case Western Reserve University (United States) 
Marinko V. Sarunic, Simon Fraser University (Canada)

Guillermo J. Tearney, Wellman Center for Photomedicine (United States)

Ruikang K. Wang, University of Washington (United States)

Maciej Wojtkowski, Nicolaus Copernicus University (Poland)

Yoshiaki Yasuno, University of Tsukuba (Japan)

\section{Session Chairs}

1 Ophthalmic New Technology I

Joseph A. Izatt, Duke University (United States)

2 Cardiac and Small Animal

James G. Fujimoto, Massachusetts Institute of Technology (United States)

3 OCT Angiography

Christoph K. Hitzenberger, Medizinische Universiteit Wien (Austria)

4 Gl/Endoscopic/Catheter

Guillermo J. Tearney, Massachusetts General Hospital (United States)

5 Novel Microscopy

Rainer Andreas Leitgeb, Medizinische Universiteit Wien (Austria)

6 OCT New Technology I

Ruikang K. Wang, University of Washington (United States)

7 Ophthalmic New Technology II

Marinko V. Sarunic, Simon Fraser University (Canada)

8 OCT New Technology II

Yoshiaki Yasuno, University of Tsukuba (Japan)

9 Elastography

Andrew M. Rollins, Case Western Reserve University (United States)

10 Polarization

Johannes F. de Boer, Vrije University Amsterdam (Netherlands)

11 Visible Light OCT

Zhongping Chen, Beckman Laser Institute and Medical Clinic (United States)

12 Novel Contrast

Valery V. Tuchin, Saratov State University (Russian Federation) 